\title{
Effect of Heat Treatment on Microstructure and Mechanical Properties of Quenching and Partitioning Steel
}

\author{
Shao-Heng Sun ${ }^{1} \cdot$ Ai-Min Zhao ${ }^{1} \cdot$ Ran Ding $^{2} \cdot$ Xiao-Gang $\mathrm{Li}^{3}$
}

Received: 20 April 2017/Revised: 22 June 2017/Published online: 13 November 2017

(C) The Chinese Society for Metals and Springer-Verlag GmbH Germany 2017

\begin{abstract}
In order to investigate the effect of microstructural characterization on the mechanical properties and retained austenite stability, a different type of quenching and partitioning steel (I-Q\&P) through intercritical annealing before the quenching and partitioning process was designed, which can realize lamellar intercritical microstructure compared to the conventional quenching and partitioning $(\mathrm{Q} \& \mathrm{P})$ process. The morphology of ferrite and martensite/retained austenite is lamellar in the I-Q\&P steel while it is equiaxed after being heat-treated by conventional Q\&P process. The I-Q\&P steel is proved to have better formability and mechanical properties than conventional Q\&P steel, which is due to the highervolume fraction of retained austenite in the I-Q\&P steel and confirmed by electron backscattering diffraction patterns and $\mathrm{X}$-ray diffraction. Furthermore, the stability of retained austenite in I-Q\&P steel is also higher than that in conventional Q\&P steel, which is investigated by tensile tests and differential scanning calorimetry.
\end{abstract}

\section{KEY WORDS: Quenching and partitioning steel; Heat treatment; Retained austenite; Stability; Activation} energy

\section{Introduction}

With the increase in the spread of environmental awareness, the application of lightweight components in automobiles is a challenge. For the reasons of economic and ecological concerns, the reduction in automobile mass is highly desired. Speer et al. [1, 2] proposed a novel

Available online at http://link.springer.com/journal/40195

Ai-Min Zhao

aimin.zhao@ustb.edu.cn

1 Collaborative Innovation Center of Steel Technology, University of Science and Technology Beijing, Beijing 100083, China

2 Key Laboratory for Advanced Materials of Ministry of Education, School of Materials Science and Engineering, Tsinghua University, Beijing 100084, China

3 New Technology Department, Beijing Electric Vehicle CO., LTD, Beijing 102606, China quenching and partitioning (Q\&P) heat treatment, causing wide interests due to their excellent properties and potential for application in automotive structural components.

Generally, the microstructure of intercritical annealed Q\&P steel microstructure is composed of ferrite, martensite, and retained austenite, and their volume fractions and distributions determine the mechanical properties. The phase fraction is controlled by annealing, athermal martensite transformation, and isothermal partitioning stage. Such an approach creates a wide variety of the microstructures and correspondingly large ranges of mechanical properties in Q\&P steel. Retained austenite is a metastable phase in Q\&P steel and can transform into the martensite under local stress and strain. This transformation provokes work hardening, which can delay the onset of necking and improve the ductility. Furthermore, the increase in specific volume caused by the transformationinduced plasticity (TRIP) effect can also help to close propagating cracks [3]. Therefore, normally the higher the volume fraction of the retained austenite, the better the 
mechanical properties of the steel. However, the final mechanical properties of steels depend not only on the volume fraction of the retained austenite, but also on its stability which can be adjusted by original microstructure prior to the heat treatment [4]. Compared to the equiaxed microstructure, the new lamellar one demonstrates a good TRIP effect and excellent mechanical properties for the higher stability of retained austenite [5].

Actually, knowledge is still limited concerning the influence of the fraction, morphology, distribution, and crystallographic texture of the retained austenite on the mechanical properties [6]. Some studies show that the stability of the retained austenite is influenced by its local chemical composition [7, 8], size [9], the phases surrounding the austenite $[10,11]$, the crystallographic orientation of the austenite with respect to the loading direction [8, 12, 13], and morphology of the austenite [10]. The stability of the retained austenite can be investigated by the mechanical methods or thermal methods. Not only the mechanical stability but also the thermal stability of the retained austenite during the $\mathrm{Q} \& \mathrm{P}$ process is also of great importance. The thermal decomposition temperature of the retained austenite is normally in the range of $300-550{ }^{\circ} \mathrm{C}$, and the activation energy is in a wide range. The activation energy of thermal decomposition of the retained austenite in $\mathrm{Fe}-0.12 \mathrm{C}-1.5 \mathrm{Mn}-0.7 \mathrm{Si}$ TRIP steel is around $212 \mathrm{~kJ} / \mathrm{mol}$ [14]. However, the activation energy values obtained for the decomposition of retained austenite tested by $\mathrm{P}$. V. Morra are in the range $135-156 \mathrm{~kJ} / \mathrm{mol}$ [15]. Moreover, the activation energy of thermal decomposition of the retained austenite in $\mathrm{Fe}-0.2 \mathrm{C}-1.87 \mathrm{Mn}-1.42 \mathrm{Si}-0.0405 \mathrm{Al}$ Q\&P steel is $221.3 \mathrm{~kJ} / \mathrm{mol}$ according to the differential scanning calorimetry (DSC) [16].

Compared to the conventional Q\&P steel, the current work studied a novel heat treatment schedule of Q\&P steel using martensitic microstructure prior to the $\mathrm{Q} \& \mathrm{P}$ process (M-Q\&P). While the conventional microstructure is equiaxed ferrite, retained austenite, and martensite, the M-Q\&P microstructure consisted of lamellar-retained austenite surrounded by recovered ferrite laths and martensite, which is produced by a three-stage heat treatment. The transformation behavior of the retained austenite in these two different Q\&P steels during uniaxial tensile testing was researched, and the activation energy of the retained austenite was investigated.

\section{Experimental}

The chemical composition of the steel investigated in the present study is given in Table 1. After forged, the steel was hot and cold rolled into sheets $1.5 \mathrm{~mm}$ thick. The austenitization starting temperature $\left(A_{1}\right)$ and austenitization finishing temperature $\left(A_{3}\right)$ calculated by Thermo-Calc based on the thermodynamic database TCFE7 are 715.7 and $836.7^{\circ} \mathrm{C}$, respectively. Before the Q\&P process, the sample with I-Q\&P heat treatment schedule was first heated to the fully austenitic region by an electric air furnace at $960{ }^{\circ} \mathrm{C}$ and held for 20 min followed by quenching in oil to acquire fully martensitic microstructure. And the two heat treatment schedules are shown in Fig. 1.

The samples for scanning electron microscopy (SEM) observation were ground, mechanically polished, and etched with $4 \%$ nital. The samples for electron backscattering diffraction (EBSD) and X-ray diffraction (XRD) were ground and then electrolytically polished with $20 \%$ perchloric acid and $80 \%$ ethanol. The microstructure was observed using a field-emission scanning electron microscope (FEI Company, Hillsboro, USA) with an EBSD unit. An accelerating voltage of $20 \mathrm{kV}$ and a step size of $0.08 \mu \mathrm{m}$ were used in EBSD scans. XRD was made by using $\mathrm{Cu}-K_{\alpha}$ radiation operating at $40 \mathrm{kV}$ and $150 \mathrm{~mA}$. The volume fraction of retained austenite was obtained by comparing the integrated intensity of the $(200)_{\gamma},(220)_{\gamma}$, $(311)_{\gamma},(200)_{\alpha}$, and $(211)_{\alpha}$ peaks [17]. The carbon concentration of the retained austenite $\left(\mathrm{C}_{\gamma}\right.$, wt $\left.\%\right)$ was calculated using the following equation [18]:

$C_{\gamma}=\left(a_{\gamma}-0.3547\right) / 0.00467$,

where $a_{\gamma}$ is the retained austenite lattice constant, $\mathrm{nm}$.

The tensile specimens with $1.5 \mathrm{~mm}$ in thickness were cut from the sheet parallel to the rolling direction by a wire-electrode machine. The gauge length was $50 \mathrm{~mm}$, and the tensile tests were performed at a speed of $1 \mathrm{~mm} / \mathrm{min}$ on a SANS XYB605C universal testing machine. The Erichsen cupping tests were performed by Zwick-BUP600 sheet forming machine and accord to GB/T4156-2007 standard.

Table 1 Chemical composition of Q\&P steel (wt\%)

\begin{tabular}{lllllll}
\hline $\mathrm{C}$ & $\mathrm{Si}$ & $\mathrm{Mn}$ & $\mathrm{P}$ & $\mathrm{S}$ & $\mathrm{Al}$ & $\mathrm{Nb}$ \\
\hline 0.23 & 1.93 & 2.18 & 0.0081 & 0.0044 & 0.043 & 0.023 \\
\hline
\end{tabular}



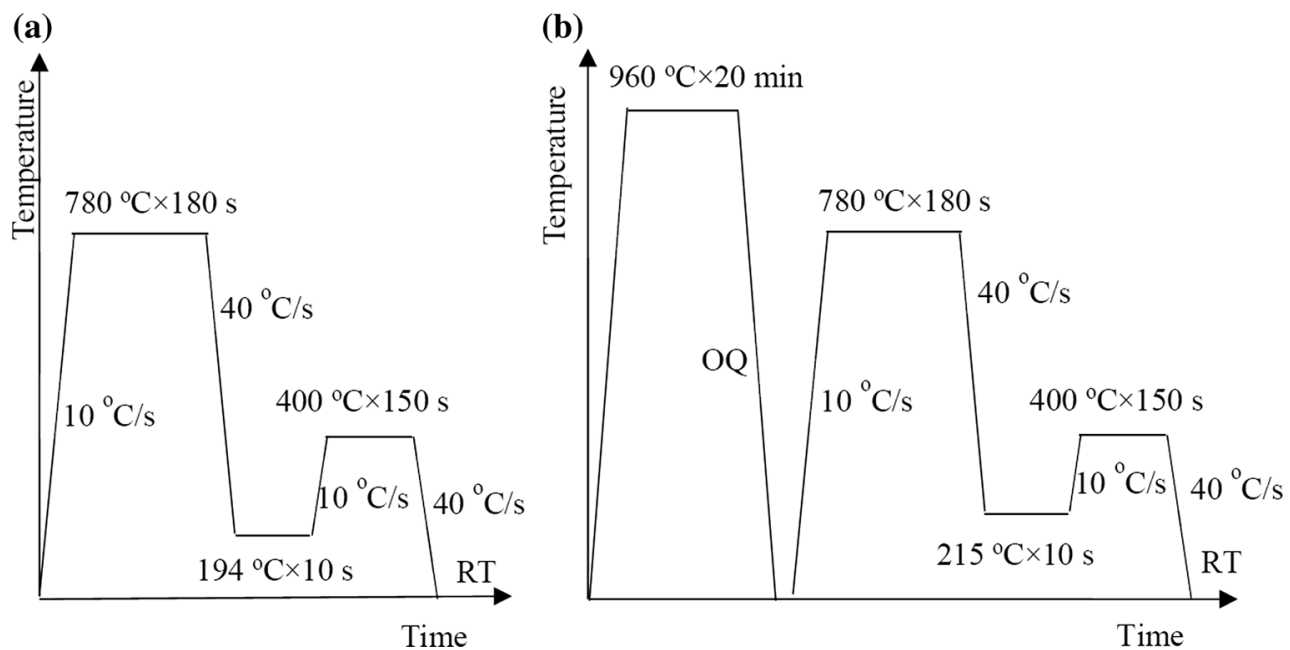

Fig. 1 Schematic diagram of heat treatments for conventional a and I-Q\&P b samples. OQ oil-quenching, RT room temperature

Differential scanning calorimetry (DSC) was used to study the retained austenite thermal stability. Specimens were heated from room temperature to $900{ }^{\circ} \mathrm{C}$ at a heating rate of $5,10,15,20 \mathrm{~K} / \mathrm{min}$. And then the activation energy $E$ of the retained austenite was calculated by Kissinger analysis [16].

\section{Result}

\subsection{Microstructures}

The initial microstructures before $\mathrm{Q} \& \mathrm{P}$ process are shown in Fig. 2. The original microstructure of the conventional Q\&P steel is the as-cold-rolled banded ferrite and pearlite, while that of the I-Q\&P steel is full martensite.

The microstructures resulted from the two heat treatments are shown in Fig. 3. In both samples, dark regions represent ferrite $(\mathrm{F})$. The tempered and untempered martensite (TM and UM) are distinguishable in both samples subject to different conditions [19]. TM was produced in the quenching process and tempered during the partitioning process, while UM was produced in the second cooling process after partitioning, without tempering. Islands of UM and the retained austenite (RA) appear to be light and smooth, and TM is easy to be etched compared with UM. By comparison with Fig. 3a, b, the conventional Q\&P steel consists of equiaxed ferrite surrounded by UM/ RA and TM band which is originated from the perlite band after cold rolling, while the I-Q\&P steel consists of acicular-retained austenite (ARA), TM, and blocky UM/RA, and the ferrite separated by UM/RA and TM is lath in shape inherited from oil-quenched martensite. What's more, the average size of UM/RA in the I-Q\&P microstructure is smaller than that in the conventional microstructure.

The volume fraction of each constituent phase and mass fraction of carbon in the retained austenite of tested steels are shown in Table 2. The volume fractions of the ferrite and tempered martensite are calculated by dilatometry based on lever rule. The volume fraction and carbon content of the retained austenite are investigated by XRD.
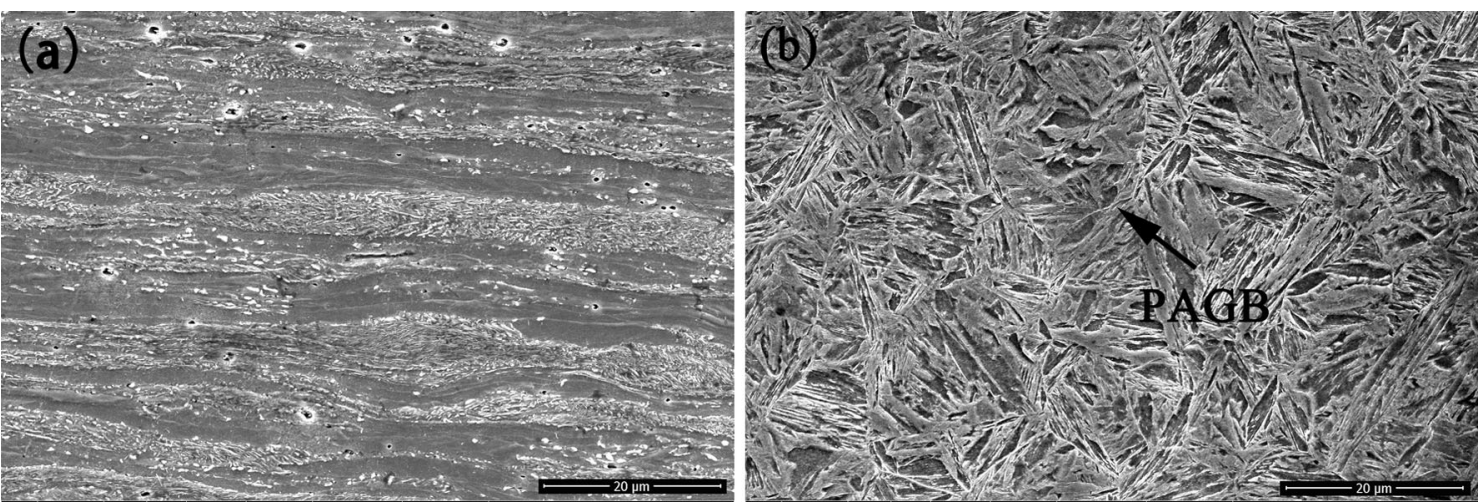

Fig. 2 Microstructure of tested steel before Q\&P process (PAGB: prior austenite grain boundary): a conventional sample; b I-Q\&P sample 


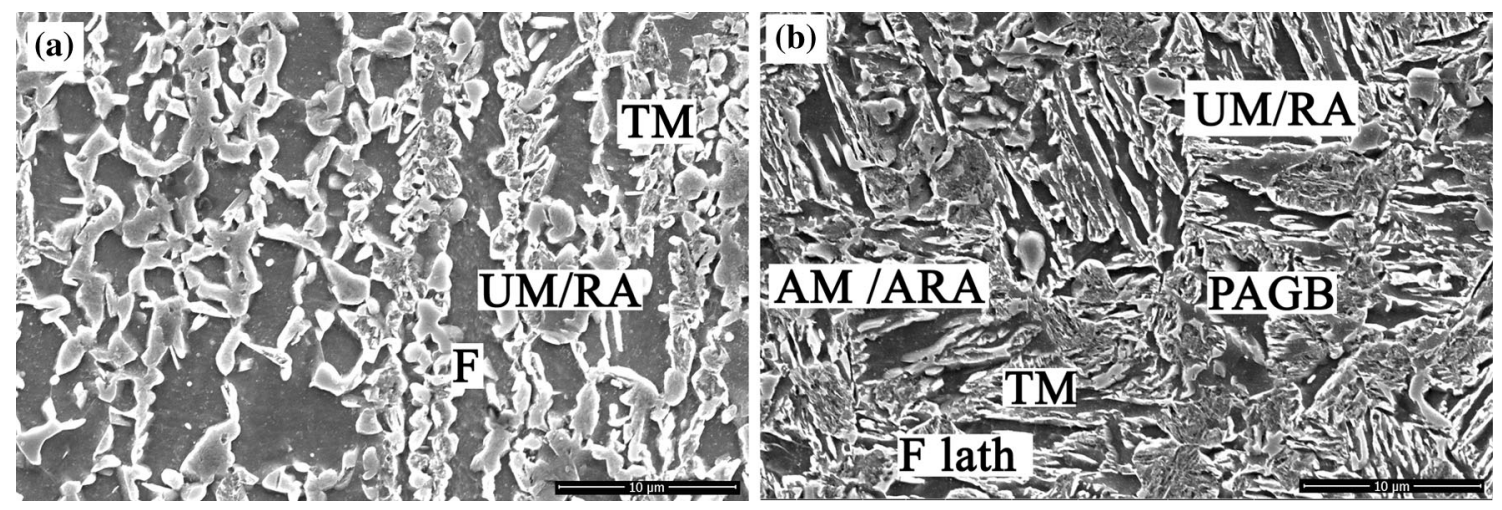

Fig. 3 SEM micrographs of conventional a and I-Q\&P b samples (PAGB, TM, UM, AM, RA, ARA, and F refer to prior austenite grain boundary, tempered martensite, untempered martensite, acicular martensite, retained austenite, acicular-retained austenite, and ferrite, respectively)

Table 2 Volume fraction of each constituent phase and mass fraction of carbon in retained austenite of tested steels

\begin{tabular}{lccccc}
\hline Sample & $f_{\mathrm{F}}(\%)$ & $f_{\mathrm{TM}}(\%)$ & $f_{\mathrm{UM}}(\%)$ & $f_{\mathrm{RA}}(\%)$ & $C_{\gamma}(\%)$ \\
\hline Conventional & 57.53 & 22.47 & 14.23 & 5.77 & 1.097 \\
I-Q\&P & 41.42 & 38.58 & 6.03 & 13.97 & 1.070
\end{tabular}

$f_{\mathrm{F}}$, volume fraction of ferrite; $f_{\mathrm{M}}$, volume fraction of tempered martensite; $f_{\mathrm{M}}$, volume fraction of untempered martensite; $f_{\mathrm{RA}}$, volume fraction of retained austenite; $C_{\gamma}$, carbon content of retained austenite

Thus, the rest is untempered martensite. It indicates that the volume fraction of $\mathrm{UM}$ in the conventional sample is higher than that in the I-Q\&P sample. Therefore, the volume fraction of RA is lower in the conventional sample. However, carbon content of the retained austenite is very similar. The initial-volume fraction of the retained austenite (RA) by calculation is equal under different quenching temperatures, so the chemical composition of the austenite is roughly equal after quenching to the target temperatures. However, there is considerable amount of RA in the conventional sample transforming to untampered martensite (UM) in the final cooling process after partitioning, and there is no enough time for the carbon diffusing and enriching to the RA from the UM. Therefore, the composition in the two samples is very similar.

\subsection{Retained Austenite Characterization}

EBSD images of the tested steels are shown in Fig. 4, in which color part corresponds to fcc phase (RA) and others to bcc phases (F, TM, and UM). The dark regions refer to UM owing to their high defect densities and large quantities of substructures that resulted in low qualities of Kikuchi bands [20]. In the conventional sample, $F$ is equiaxed, and almost all of the RA are present as discrete particles between the ferrite grains and located in close vicinity of UM. Moreover, TM, UM, and RA in banded distribution correspond to the distribution of pearlite in the conventional original microstructure, because austenite preferentially nucleates in the pearlite region during the process of intercritical annealing. It has been proven that the presence of UM has a negative influence on this austenite transformation stability due to its constraining effect on the strain distribution [6]. Thus, the existence of banded structure reduces the stability of RA. In the I-Q\&P sample, $\mathrm{F}$ is lamellar, and RA is mainly acicular between the ferrite laths and/or martensite laths. The UM islands located near the white line confirm that blocky RA near the prior austenite boundaries has a lower stability and easily transforms into the martensite during the second cooling process after partitioning [21]. Some acicular RA are too small to be resolved by the restriction of EBSD resolution, but they could not have a great influence on the total stability and other properties for the very little-volume fraction of them. Actually, RA in the micrograph is less than that in the actual sample. Blondé et al. [8] have demonstrated that the RA with the $\{111\}$ component is the most stable, and the stability of RA with the $\{200\}$ component is the worst. It is obvious that the volume fraction of RA with the $\{111\}$ component in the I-Q\&P sample is much more than that in the conventional sample. Therefore, the stability of RA in the I-Q\&P sample is more stable.

The volume fractions of RA at strains of $0,0.02,0.05$, 0.07 , and fracture are investigated by XRD as shown in Fig. 5. The result shows that the volume fraction of RA decreases with the increase in deformation due to the occurrence of strain-induced transformation which can enhance the mechanical properties and delay fracture. But the decreasing tendency of RA is different. The volume fraction change of the conventional sample is about linear from the initial stage of the deformation to fracture. Whereas, the volume fraction change of the I-Q\&P sample is similar at the initial stage ( $<5 \%$ engineering strain) of 

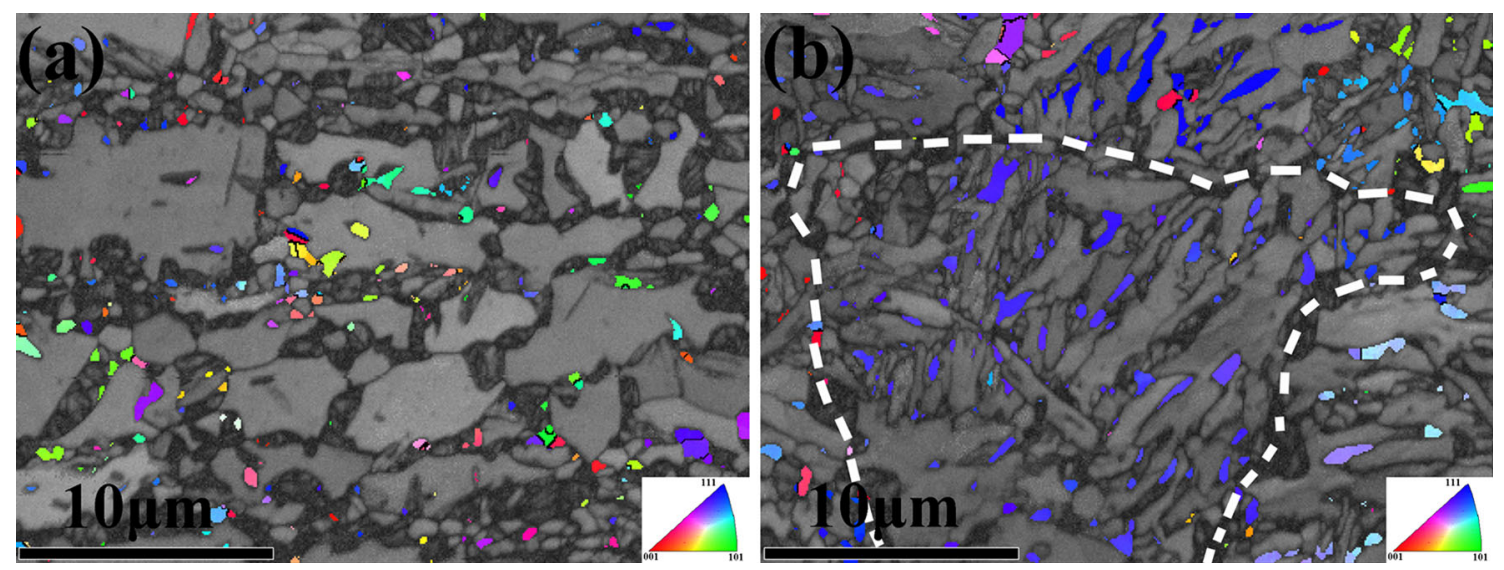

Fig. 4 EBSD images of conventional a and I-Q\&P b samples with the color region representing retained austenite

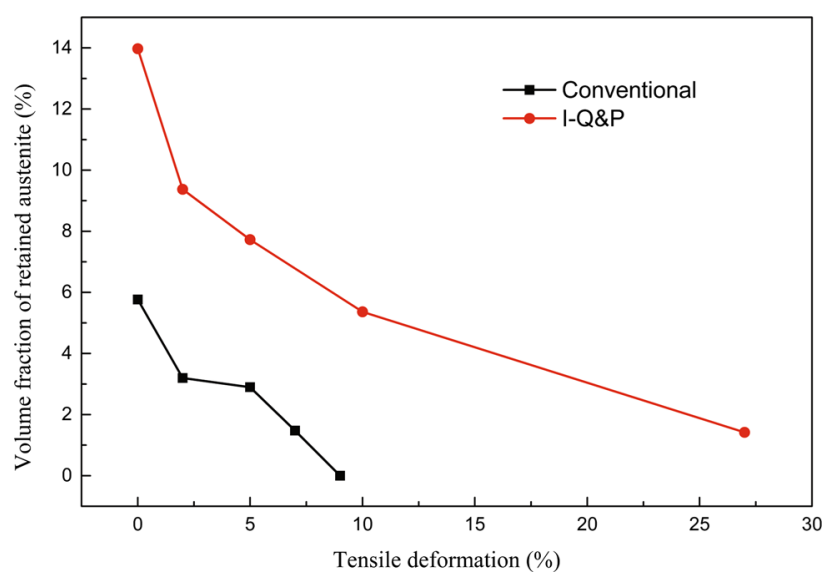

Fig. 5 Volume fraction of retained austenitic as a function of tensile deformation for tested steels

the deformation, and then the decrease becomes gentle until nearing the fracture, which demonstrates that the mechanical stability of RA in I-Q\&P sample is different from the stability in the conventional sample.

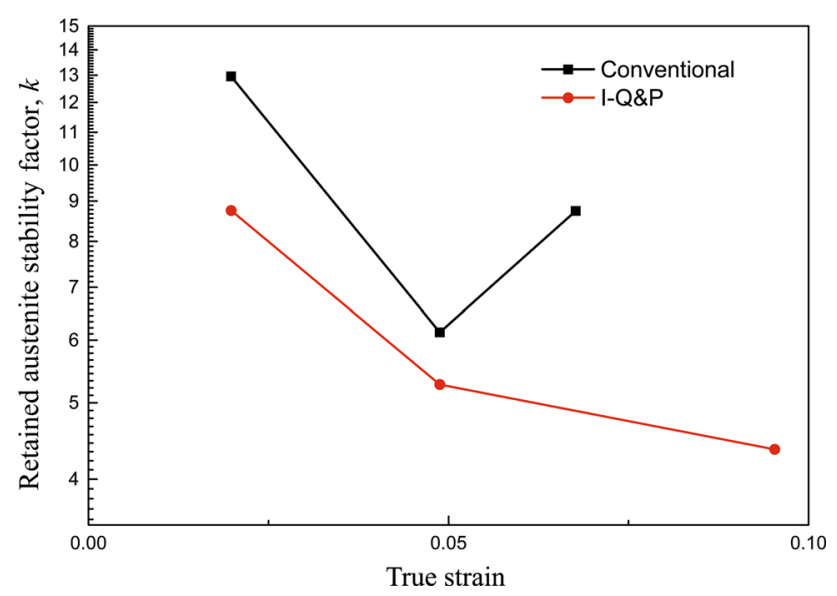

Fig. 6 Variations of $k$ values as a function of true strain in tested steels
Figure 6 shows variations of retained austenite stability factor $k$ as a function of true strain $\varepsilon$ which is between $0 \%$ and fracture in conventional and I-Q\&P steels. $k$ is defined by the following equation [22]:

$\lg f_{\gamma}=\lg f_{\gamma_{0}}-k \varepsilon$

where $f_{\gamma}$ and $f_{\gamma O}$ are the volume fractions of RA after and before deformation, respectively. The smaller the $k$ value, the more stable the RA is. It can be seen that RA in the I-Q\&P steel has higher austenite stability than that in conventional steel.

Table 2 indicates that the I-Q\&P sample has a higher initial-volume fraction of RA than the conventional sample, although the volume fraction of RA calculated is equal under different quenching temperatures. This is because considerable amount of RA in the conventional sample transforms to UM in the final cooling process after partitioning, which does not occur obviously in the I-Q\&P sample, which is confirmed by dilatometry as shown in Fig. 7 and marked by red arrow. It indicates that the stability of RA in the I-Q\&P steel is higher than that in the conventional sample during the cooling process after partitioning.

Fresh martensite grains limit the strain accommodating capacity of the tempered martensite. This constraining effect gives rise to stress concentrations which, in turn, cause the austenite to transform at lower elongations.

In order to further research the stability of RA, thermal decomposition of RA was investigated by DSC, and the results established by heating with four different heating rates are presented in Fig. 8. It indicates that the decomposition temperature of RA in the I-Q\&P sample is higher than that in the conventional sample at any heating rate. The activation energy $E$ for austenite decomposition is determined by Kissinger analysis according to the following equation [16]: 

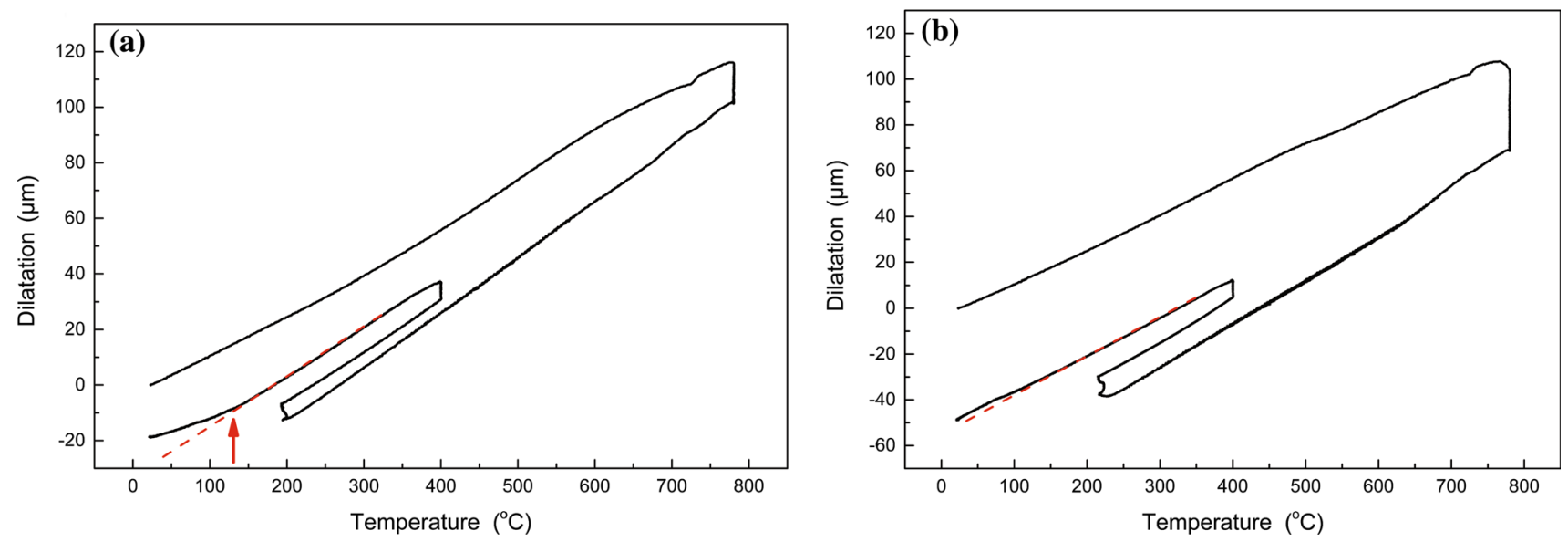

Fig. 7 Dilatometry curves for conventional a and I-Q\&P b samples after different heat treatments
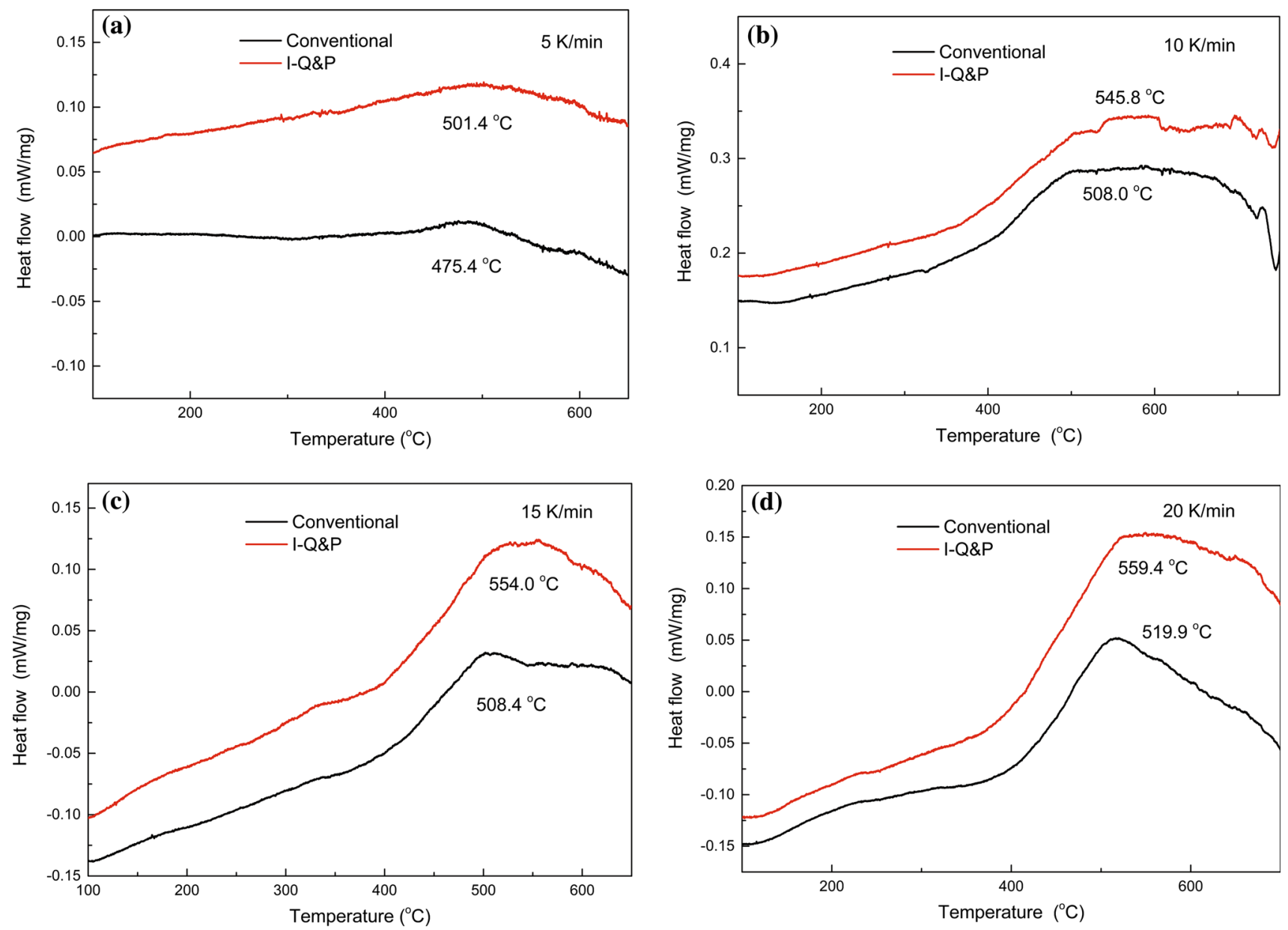

Fig. 8 Heat flow of tested steels as a function of temperature at heating rates of $5 \mathrm{~K} / \mathrm{min} \mathbf{a}, 10 \mathrm{~K} / \mathrm{min} \mathbf{b}, 15 \mathrm{~K} / \mathrm{min} \mathbf{c}, 20 \mathrm{~K} / \mathrm{min} \mathbf{d}$

$\ln \left(\frac{T^{2}}{\Theta}\right) \approx \frac{E}{R T}+\ln \left(\frac{E}{R K_{0}}\right)$,

where $T$ is the temperature of austenite decomposition, $\Theta$ is the heating rate, $R$ is the universal gas constant, and $K_{0}$ is the pre-exponential factor. Thus, the activation energy $E$ is the product of $R$ and slope of $\ln \left(T^{2} / \Theta\right)$ vs. $1 / T$.

Figure 9 shows the plots of $\ln \left(T^{2} / \Theta\right)$ vs. $1 / T$ for the austenite decomposition peaks, and the obvious spoiled points are picked out. The activation energy of RA 


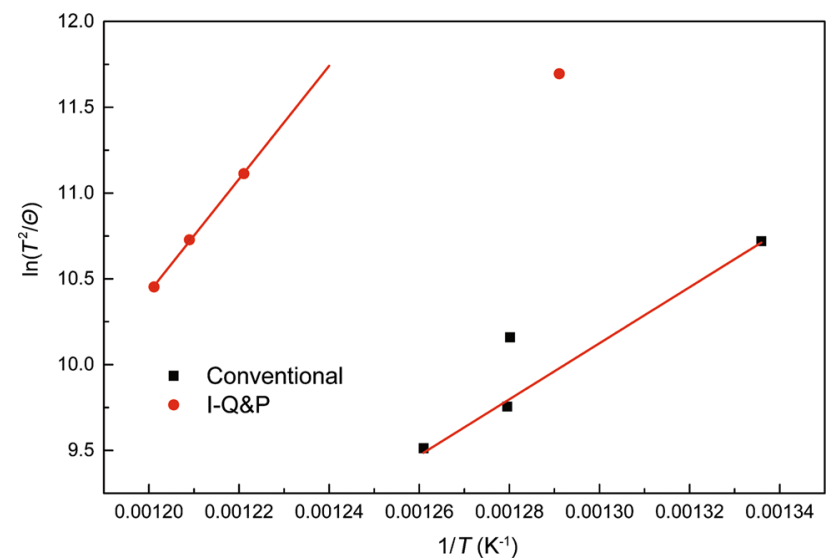

Fig. 9 Kissinger analysis $\left(\ln \left(T^{2} / \Theta\right)\right.$ vs. $\left.1 / T\right)$ for determination of activation energy of retained austenite decomposition

decomposition in the conventional sample is determined to be $135.858 \mathrm{~kJ} / \mathrm{mol}$, while the activation energy in the I-Q\&P sample is $274.312 \mathrm{~kJ} / \mathrm{mol}$, which is suggested that the thermal stability of RA in the I-Q\&P sample is more than that in the conventional sample.

\subsection{Mechanical Properties}

The engineering stress-strain curves are presented in Fig. 10, and the mechanical properties are summarized in Table 3. Continuous yielding is observed in all samples, which is usually associated with high mobile dislocation density. The curves also indicate that there is no local necking in the conventional sample, but an obvious local necking occurs for the I-Q\&P sample. The yield ratio of both samples is below 0.5 . Compared with the conventional sample, in spite of relatively lower tensile strength, a large elongation is obtained in the I-Q\&P sample. The I-Q\&P sample also produces a higher product of strength and elongation (more than $31 \mathrm{GPa} \%$ ), which indicated that the I-Q\&P steel with lamellar microstructure has a better

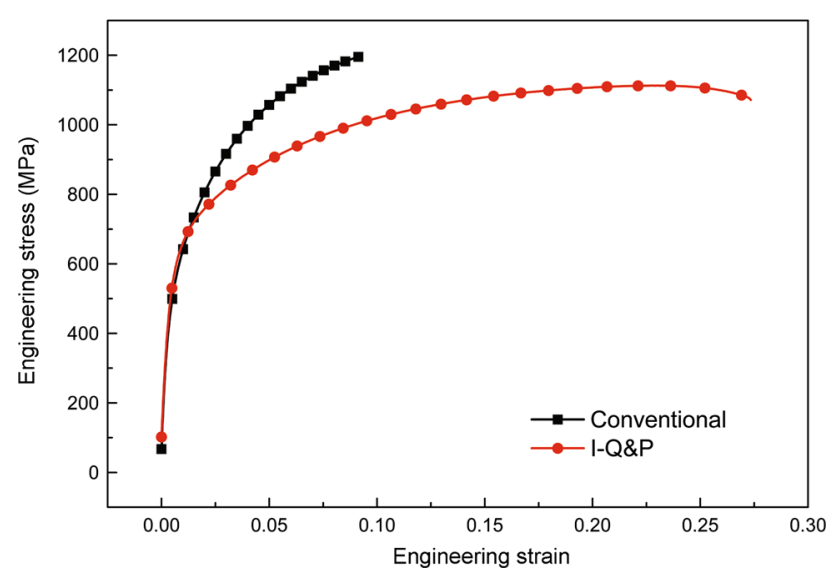

Fig. 10 Engineering stress-strain curves of tested steels combination of the strength and ductility, and more energy could be absorbed during crash.

The excellent mechanical properties of the I-Q\&P steel are attributed to the higher-volume fraction and stability of RA and the fine grain sizes of the ferrite laths. The stability of RA is attributed to not only carbon concentration but also its morphology [23]. Acicular- or lath-retained austenite is more stable than blocky RA [24], although it has lower carbon content [25]. During the process of tensile testing, the acicular RA shows better ability to resist TRIP effect than blocky RA, so the stress concentration could be effectively relieved due to the transformation-induced plasticity effect of interlath-retained austenite [4]. Moreover, when the microstructure consists of fine ferrite and martensite, the development of cracks needs to pass through more interface, which can delay the fracture, and the uniform deformation is prolonged. Thus, the excellent mechanical properties can be accessible easily to the I-Q\&P sample.

\subsection{Formability}

The cupping force-travel curves are presented in Fig. 11. The Erichsen index (EI) is widely used as an indication of formability for sheet materials. The higher the EI value, the better the formability. The EI of I-Q\&P sample is $8.444 \mathrm{~mm}$, while that of conventional sample is $5.753 \mathrm{~mm}$. For the conventional sample, the slope of the curve generally remains constant at the region close to the fracture, which is in an instant without any local necking. While in the I-Q\&P sample, the slope of the curve gradually becomes small, which demonstrates that fracture comes up slowly with obvious local necking. So the I-Q\&P sample has a better fracture toughness than the conventional sample. The photos of samples after Erichsen cupping testing are also shown in the figure. In the conventional sample, cracks are long and wide and show a multi-directional development trend. There is a crack developing along the cupping edge and resulting that the arrow shown region is easy to fall off. The sample is damaged severely. However, in the I-Q\&P sample, there is only a small and short crack. Therefore, the formability of the I-Q\&P steel is expected to be better than the conventional steel.

The instantaneous strain-hardening exponent $n$ is also widely used as an indication of formability of sheet materials. More formable materials will exhibit higher $n$ values that are maintained to a higher level of strain. The Hollomon equation describes the relationship between the true stress and true strain and is commonly used to study the strain-hardening behavior. It is expressed as:

$\sigma=\Gamma \varepsilon^{n}$,

where $\Gamma$ is the strength coefficient. If the experimental data 
Table 3 Mechanical properties of tested steels after Q\&P treatment

\begin{tabular}{lllllr}
\hline Sample & $R_{0.2}(\mathrm{MPa})$ & $R_{\mathrm{m}}(\mathrm{MPa})$ & YR & $A(\%)$ & $R_{\mathrm{m}} \times A(\mathrm{GPa} \%)$ \\
\hline Conventional & 517.99 & 1197.61 & 0.4325 & 9.5 & 11.77295 \\
I-Q\&P & 541.98 & 1112.83 & 0.4870 & 28.0 & 31.15924 \\
\hline
\end{tabular}

$R_{\mathrm{m}}$, tensile strength; $R_{0.2}$, yield strength; YR, yield ratio; $A$, total elongation; $R_{\mathrm{m}} \times A$, product of tensile strength and total elongation

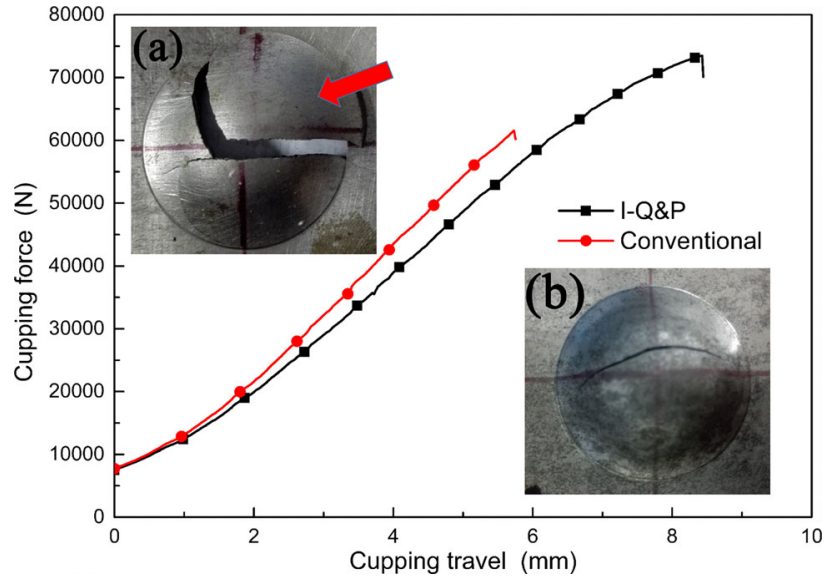

Fig. 11 Cupping force-travel curves and photos of conventional a and I-Q\&P b samples after Erichsen testing

satisfy the Hollomon equation and the curve is plotted on logarithmic coordinates, then a linear regression with slope $n$ can be determined. So $n$ is defined as:

$n=\frac{\mathrm{d}(\ln \sigma)}{\mathrm{d}(\ln \varepsilon)}$,

Figure 12 plots the $n$ values as a function of true strain for the tested steels. The straight line corresponds to the instability criterion $n=\varepsilon_{\mathrm{u}}$, where $\varepsilon_{\mathrm{u}}$ is the true strain at the beginning of necking. It indicates that the conventional sample fractures before local necking, so the I-Q\&P steel

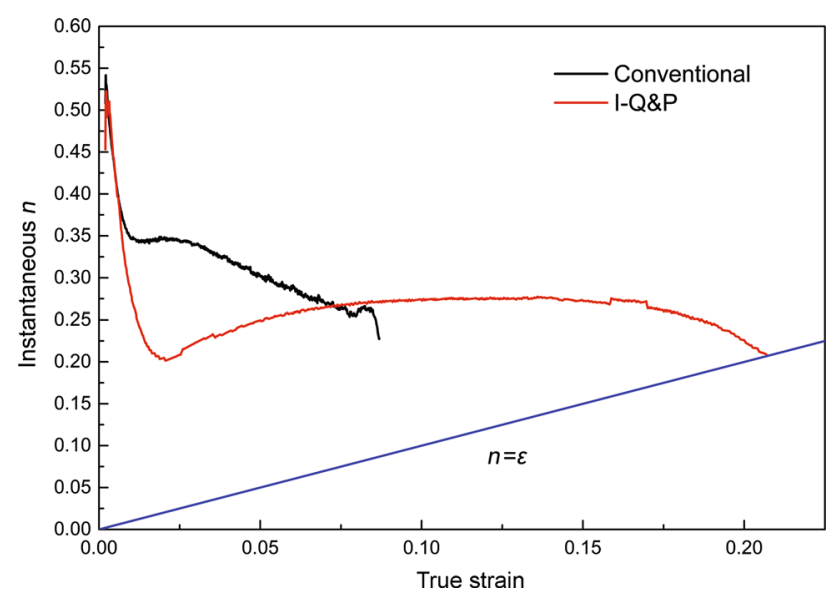

Fig. 12 Instantaneous work-hardening exponent $n$ for conventional and I-Q\&P samples as a function of true strain has a better ductility. Moreover, the conventional sample has a high maximum $n$ value of about 0.35 , and this value gradually drops as the sample is deformed. The $n$ value of I-Q\&P sample first declines rapidly and then slowly rises and slowly declines. It reaches a peak value about 0.28 and exhibits sustained values at larger strains.

The observed difference in work-hardening behavior can be explained in terms of different volume fractions and stability of RA in the two samples [26]. The volume fraction and stability of RA in the I-Q\&P sample is higher than that in the conventional sample. Therefore, the consumption rate of RA in the I-Q\&P sample is lower than that in the conventional sample, which allows for the instantaneous $n$ value to increase at higher strains compared to the conventional sample. Thus, local necking is suppressed, and the I-Q\&P steel obtains excellent formability.

\subsection{Fracture Mechanisms}

Fracture morphologies of the tested steels are shown in Fig. 13. The fracture surface of the conventional sample is primarily composed of cleavage facets and dimples (Fig. 13a), while the fracture surface of the I-Q\&P sample contains mainly dimples which is a mark of admirable fracture toughness (Fig. 13b). On the one hand, the fracture toughness depends primarily on the volume fractions of softer phases and interface (grain boundary and phase boundary). RA and F are typical softer phase. Besides, the TM which undergoes carbon depletion and recovery during partitioning process is more ductile and hence easily accommodate the deformation compared with the UM [27]. As shown in Table 2, the volume fraction sum of RA, F, and TM in the I-Q\&P sample is greater than that in the conventional sample. Moreover, the volume fraction of the interface is higher in the I-Q\&P sample due to its fine grain size. On the other hand, the fracture toughness is affected by the morphology and distribution of the martensite [28]. The crack propagates along the banded martensite during the process of fracture, so the banded martensite easily results in the cleavage fracture mechanism [29]. As shown in Fig. 6, the conventional sample mainly consists of banded martensite and blocky ferrite, while there is no banded martensite in the I-Q\&P sample. Consequently, the fracture toughness of the $\mathrm{M}-\mathrm{Q} \& \mathrm{P}$ sample is relatively better than that of the conventional sample. 

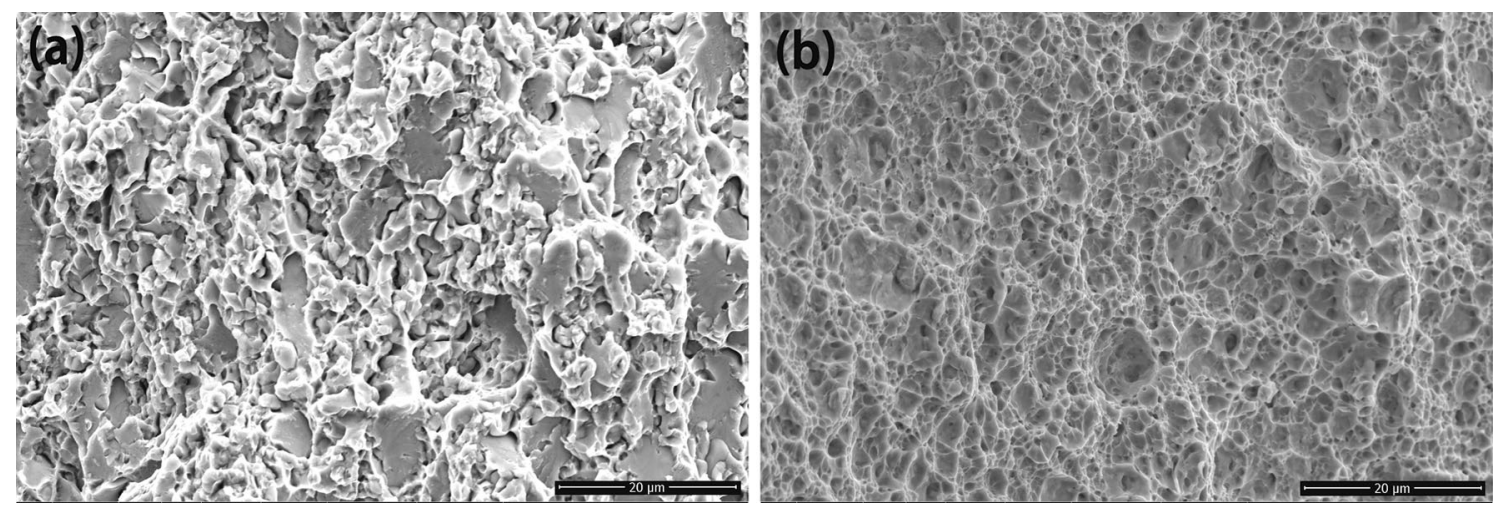

Fig. 13 SEM micrographs of fracture surfaces of conventional a and I-Q\&P $\mathbf{b}$ samples

\section{Conclusion}

1. Two distinct Q\&P steel microstructures were produced via multistage heat treatments: conventional and I-Q\&P. In the conventional sample, the ferrite and martensite/retained austenite are blocky or banded, whereas they are lamellar in the I-Q\&P sample.

2. Retained austenite in the I-Q\&P sample is more stable than that in the conventional sample. Compared to the conventional sample, most retained austenite in the I-Q\&P sample is not only blocky in prior austenite grain boundaries but also acicular between the ferrite laths and/or martensite laths. Considerable amount of the retained austenite in the conventional sample transforms to fresh martensite in the second quenching process after partitioning, so the I-Q\&P sample has a higher-volume fraction of the retained austenite. The activation energy of the retained austenite decomposition in the conventional sample is determined to be $135.858 \mathrm{~kJ} / \mathrm{mol}$, while the activation energy in the I-Q\&P sample is $274.312 \mathrm{~kJ} / \mathrm{mol}$.

3. The I-Q\&P sample has better mechanical properties than the conventional sample. Compared with the conventional sample, in spite of relatively lower tensile strength, a large elongation is obtained in the I-Q\&P sample. The I-Q\&P sample also has a higher product of strength and elongation (more than 31 GPa\%). The I-Q\&P sample exhibits better formability and fracture toughness than the conventional sample.

\section{References}

[1] J. Speer, D.K. Matlock, B.C. De Cooman, J.G. Schroth, Acta Mater. 51, 2611 (2003)

[2] J. Speer, D. Edmonds, F. Rizzo, D. Matlock, Curr. Opin. Solid State Mater. Sci. 8, 219 (2004)

[3] J. Chiang, B. Lawrence, J.D. Boyd, A.K. Pilkey, Mater. Sci. Eng. A 528, 4516 (2011)
[4] R. Ding, D. Tang, A. Zhao, R. Dong, J. Cheng, Mater. Manuf. Process. 29, 704 (2014)

[5] A. Mark, M. Westphal, D. Boyd, J. Mcdermid, D. Embury, Can. Metall. Q. 48, 237 (2009)

[6] D. De Knijf, R. Petrov, C. Föjer, L.A.I. Kestens, Mater. Sci. Eng. A 615, 107 (2014)

[7] N. Vandijk, A. Butt, L. Zhao, J. Sietsma, S. Offerman, J. Wright, S. Vanderzwaag, Acta Mater. 53, 5439 (2005)

[8] R. Blondé, E. Jimenez-Melero, L. Zhao, J.P. Wright, E. Brück, S. van der Zwaag, N.H. van Dijk, Acta Mater. 60, 565 (2012)

[9] E. Jimenez-Melero, N.H. van Dijk, L. Zhao, J. Sietsma, S.E. Offerman, J.P. Wright, S. van der Zwaag, Acta Mater. 55, 6713 (2007)

[10] I.B. Timokhina, P.D. Hodgson, E.V. Pereloma, Metall. Mater. Trans. A 35, 2331 (2004)

[11] P.J. Jacques, F. Delannay, J. Ladrière, Metall. Mater. Trans. A 32, 2759 (2001)

[12] O. Muránsky, P. Šittner, J. Zrník, E.C. Oliver, Acta Mater. 56, 3367 (2008)

[13] S.O. Kruijver, L. Zhao, J. Sietsma, S.E. Offerman, N.H. Van Dijk, J. Phys. IV 104, 499 (2003)

[14] S. Wen, L.I. Lin, B.C.D. Cooman, P. Wollants, C.X. Yang, J. Iron Steel Res. Int. 15, 61 (2008)

[15] P.V. Morra, A.J. Böttger, E.J. Mittemeijer, J. Therm. Anal. Calorim. 64, 905 (2001)

[16] R.M. Wu, L. Wang, X.J. Jin, Phys. Proc. 50, 8 (2013)

[17] H. Maruyama, J. Jpn. Soc. Heat Treat. 17, 198 (1977)

[18] D.J. Dyson, B. Holmes, J. Iron Steel Inst. 208, 469 (1970)

[19] M.J. Santofimia, L. Zhao, J. Sietsma, Metall. Mater. Trans. A 40, 46 (2009)

[20] M.J. Santofimia, L. Zhao, R. Petrov, J. Sietsma, Mater. Charact. 59, 1758 (2008)

[21] R. Ding, D. Tang, A. Zhao, Scr. Mater. 88, 21 (2014)

[22] H.X. Yin, A.M. Zhao, Z.Z. Zhao, X. Li, S.J. Li, H.J. Hu, W.G. Xia, Int. J. Miner. Metall. Mater. 22, 262 (2015)

[23] R. Ding, D. Tang, A. Zhao, H. Guo, J. He, C. Zhi, Mater. Des. 87, 640 (2015)

[24] A.S. Podder, I. Lonardelli, A. Molinari, H.K.D.H. Bhadeshia, Proc. R. Soc. A 467, 3141 (2011)

[25] X.C. Xiong, B. Chen, M.X. Huang, J.F. Wang, L. Wang, Scr. Mater. 68, 321 (2013)

[26] J. Zhang, H. Ding, R.D.K. Misra, C. Wang, Mater. Sci. Eng. A 611, 252 (2014)

[27] W.J. Dan, Z.Q. Lin, S.H. Li, W.G. Zhang, Mater. Sci. Eng. A 552, 1 (2012)

[28] H. Ghassemi-Armaki, R. Maaß, S.P. Bhat, S. Sriram, J.R. Greer, K.S. Kumar, Acta Mater. 62, 197 (2014)

[29] Y.G. Deng, H.S. Di, J.C. Zhang, Acta Metall. Sin. (Engl. Lett.) 28, 1141 (2015) 